\title{
Die prähistorische Kupfermetallurgie - allgemeine Betrachtungen
}

\author{
Roland Haubner \\ Technische Universität Wien, Wien, Österreich \\ Eingegangen 22. Oktober 2020; angenommen 29. Oktober 2020; online publiziert 24. November 2020
}

Zusammenfassung: Kupfer ist das erste vom Menschen auf metallurgischem Weg hergestellte Metall, und somit handelt es sich dabei auch um den Beginn der Metallurgie. Die Archäometallurgie hat es sich zur Aufgabe gemacht, aus archäologischen Fundstücken die prähistorischen, metallurgischen Prozesse zu rekonstruieren. Dafür werden moderne Methoden der chemischen Analyse und auch metallographische Verfahren zur Gefügeanalyse herangezogen. Aufgrund der Komplexität der Kupfermetallurgie, beginnend bei unterschiedlichen Erzen, gefolgt von verschiedenen Verhüttungsprozessen mit unterschiedlichen Schlacken und Kupferlegierungen, bis zu den Kupfergegenständen, ist die Rückverfolgung von Prozessketten durch Auswertung von analytischen Daten sehr schwierig. Um auf dem Gebiet der Archäometallurgie Fortschritte zu erzielen, sind sicher noch viele weitere naturwissenschaftliche Untersuchungen notwendig, wobei bei der Datenauswertung der "narrative" Charakter der Archäologie im Hintergrund und der "naturwissenschaftliche" Charakter der Metallurgie im Vordergrund stehen muss.

Schlüsselwörter: Kupfer, Schlacke, Bronze, Verhüttung

Prehistoric Copper Metallurgy-General Considerations

Abstract: Copper is the first metal produced by humans in a metallurgical way and thus marks the beginning of metallurgy. Archaeometallurgy has set itself the task of reconstructing prehistoric, metallurgical processes from archaeological finds. For this purpose, modern methods of

Diesen Artikel widme ich Herrn emer. o. Univ. Prof. Dipl.-Ing. Dr. Roland Stickler, der mir bereits in meiner Jugend die wesentlichen Aspekte für wissenschaftliches Arbeiten vermittelt hat.

Prof. Dr. R. Haubner ( $\triangle$ )

Technische Universität Wien,

Getreidemarkt 6/164-03,

1060 Wien, Österreich

roland.haubner@tuwien.ac.at chemical analysis and metallographic methods for structural analysis are used.

Due to the complexity of copper metallurgy, starting with different ores, followed by different smelting processes with different slags and copper alloys, up to the copper objects, it is very difficult to trace process chains by evaluating analytical data.

In order to achieve progress in the field of archeometallurgy, many more scientific investigations are necessary, with the "narrative" character of archeology in the background and the "scientific" character of metallurgy in the foreground of the data evaluation.

Keywords: Copper, Slag, Bronze, Smelting

\section{Archäometallurgie des Kupfers}

Die Metallurgie des Kupfers stand am Beginn der Metallurgie und entwickelte sich von der Kupfer- über die Bronzezeit bis zu den modernen Verfahren. Es ist davon auszugehen, dass zuerst die einfachen Verfahren der Kupfergewinnung eingesetzt wurden und mit zunehmender Erfahrung auch kompliziertere metallurgische Prozesse entwickelt wurden. Dies lässt sich aus den archäologischen Funden und den archäometallurgischen Untersuchungen gut nachvollziehen [1-4].

Auch wenn die Archäologie zu den „narrativen Wissenschaften" zählt, sind bei der Interpretation der Ergebnisse die grundlegenden naturwissenschaftlichen Fakten zu beachten.

Es ist z. B. nicht anzunehmen, dass in der Steiermark die chemischen Reaktionen der Metallurgie anders ablaufen als im Rest der Welt. Ebenfalls als unwahrscheinlich gilt, dass sich die Grundlagen der Chemie zwischen Bronze- und Neuzeit verändert haben.

Weiters sollte man vermeiden, sich den bronzezeitlichen Metallurgen mit dem heutigen Wissensstand der Forschung vorzustellen. Diese Menschen hatten keinerlei analytische Methoden zur Qualitätskontrolle. Es ist viel- 
mehr anzunehmen, dass Rituale zur Metallgewinnung durch mündliche Überlieferung weitergegeben wurden.

Ein ganz entscheidender Punkt, der zu Fehlinterpretationen von chemischen oder physikalischen Resultaten führen kann, ist, dass die gewählte Analysentechnik nicht für die vorliegende Problemstellung geeignet ist. Wenn eine ungeeignete Analysenmethode eingesetzt wird, die methodisch richtige Ergebnisse liefert, sind das erhaltene Endergebnis und die daraus erhaltenen Schlussfolgerungen einfach falsch.

Auf bereits zahlreich vorhandene Literaturverweise wird an dieser Stelle verzichtet, und ich möchte im Folgenden die bronzezeitliche Metallurgie des Kupfers zusammenfassen.

\section{Die Erze zur Kupfergewinnung}

Während der Kupferzeit von etwa 5000 bis 3000 v. Chr. könnte bei den metallurgischen Handwerksmeistern etwa folgende mündliche Werkanleitung bestanden haben: „Such die bunten Steine und sammle sie ein. Mach diese bunten Steine im Feuer so heiß, bis das rote unzerbrechliche Material entsteht." Dies wäre die Anleitung zur Kupfergewinnung aus oxydischen Erzen, wie z. B. Cuprit $\left(\mathrm{Cu}_{2} \mathrm{O}\right)$ und Malachit $\left(\mathrm{Cu}_{2}(\mathrm{OH})_{2}\left(\mathrm{CO}_{3}\right)\right)(\mathrm{Abb} .1 \mathrm{a}, \mathrm{b})$.

In weiterer Folge wurden bevorzugt Fahlerze eingesetzt, die neben $\mathrm{Cu}$ noch $\mathrm{Fe}$, Sb und As enthalten. Die wichtigsten Minerale sind Tetraedrit $(\mathrm{Cu}, \mathrm{Fe})_{12} \mathrm{Sb}_{4} \mathrm{~S}_{13}(\mathrm{Abb} .1 \mathrm{c})$ und Tennantit $(\mathrm{Cu}, \mathrm{Fe})_{12} \mathrm{As}_{4} \mathrm{~S}_{13}$, welche selbst nicht bunt sind, aber aufgrund des Verwitterungsprodukts Malachit leicht zu finden waren.

Das mengenmäßig wichtigste Kupfermineral ist der Chalkopyrit (CuFeS 2 ) (Abb. 1d).
Analysen von Kupferprodukten zeigten, dass Fahlerz zeitlich vor Chalkopyrit verarbeitet wurde [5].

\section{Die Kupfergewinnung aus den vorhande- nen Erzen}

Oxidische Kupfererze lassen sich leicht mit Kohlenstoff zu metallischem $\mathrm{Cu}$ reduzieren. Dies kann einfach in einem Tiegel oder Herd im Holzkohlefeuer erfolgen. Von der Dauer des Reduktionsschritts ist es abhängig, wieviel Sauerstoff im $\mathrm{Cu}$ gelöst bleibt und somit wieviel $\mathrm{Cu}_{-} \mathrm{Cu}_{2} \mathrm{O}$ Eutektikum gebildet wird. Durch das Vorliegen eines Eutektikums kann bereits beim Schmelzpunkt von $\mathrm{Cu}\left(1085^{\circ} \mathrm{C}\right)$ eine vollständig aufgeschmolzene Legierung erhalten werden (Abb. 2a, b). Aus der Mondseekultur der späten Jungsteinzeit sind $\mathrm{Cu}$ Gegenstände mit $\mathrm{Cu}-\mathrm{Cu}_{2} \mathrm{O}$ Eutektikum im Gefüge bekannt [6-8].

Eine vollständige Entfernung von Sauerstoff aus der Schmelze war zu dieser Zeit nicht möglich, da geeignete Desoxidationsmittel, wie z.B. elementarer Phosphor, nicht zur Verfügung standen. Das heute industriell eingesetzte „Polen“ mit feuchten Holzstämmen könnte jedoch durch Umrühren mit frischen Holzstäbchen eingesetzt worden sein, um bessere Materialeigenschaften zu erhalten.

Die Fahlerze als Erzbasis erfordern bereits einen zweistufigen Prozess, um metallisches $\mathrm{Cu}$ herzustellen. Aus chemischer Sicht müssen die Elemente $\mathrm{S}$, As sowie $\mathrm{Sb}$ oxidiert und das $\mathrm{Cu}$ reduziert werden. Thermodynamische $\mathrm{Be}-$ rechnungen zeigten, dass mit Kohlenstoff (Holzkohle) keine Reaktion stattfindet, denn $\mathrm{C}$ reagiert mit $\mathrm{O}$ zu $\mathrm{CO}$ oder $\mathrm{CO}_{2}$, aber es erfolgt keine Reaktion mit den Fahlerzen. Um Schwefel aus dem Erz zu entfernen, ist der Prozessschritt
Abb. 1: Fotosvon Kupfererzen: a Cuprit, Demokratische Republik Kongo, b Malachit, Demokratische Republik Kongo, c Tetraedrit, Frankreich, d Chalkopyrit, China
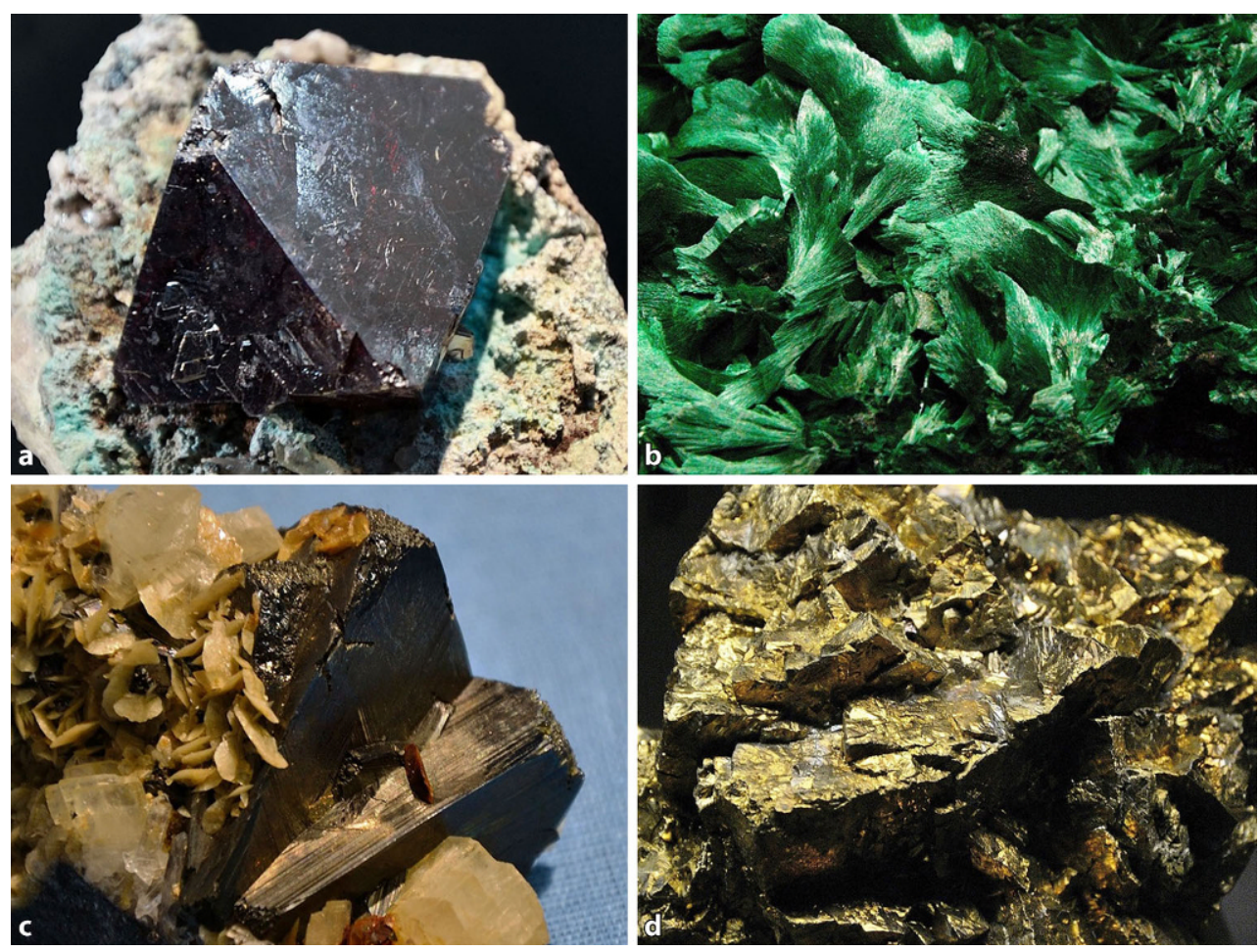
Abb. 2: Gefüge von Kupferlegierungen nach der Gewinnung aus unterschiedlichen $\mathrm{Er}$ zen im Lichtmikroskop (LOM): a, b $\mathrm{Cu}-\mathrm{Cu}_{2} \mathrm{O}$ aus oxydischen Erzen, c, d Cu-Cu3As aus Fahlerzen, e, $\mathrm{f} C \mathrm{Cu}-\mathrm{Cu}_{2} \mathrm{~S}$ aus Chalkopyrit
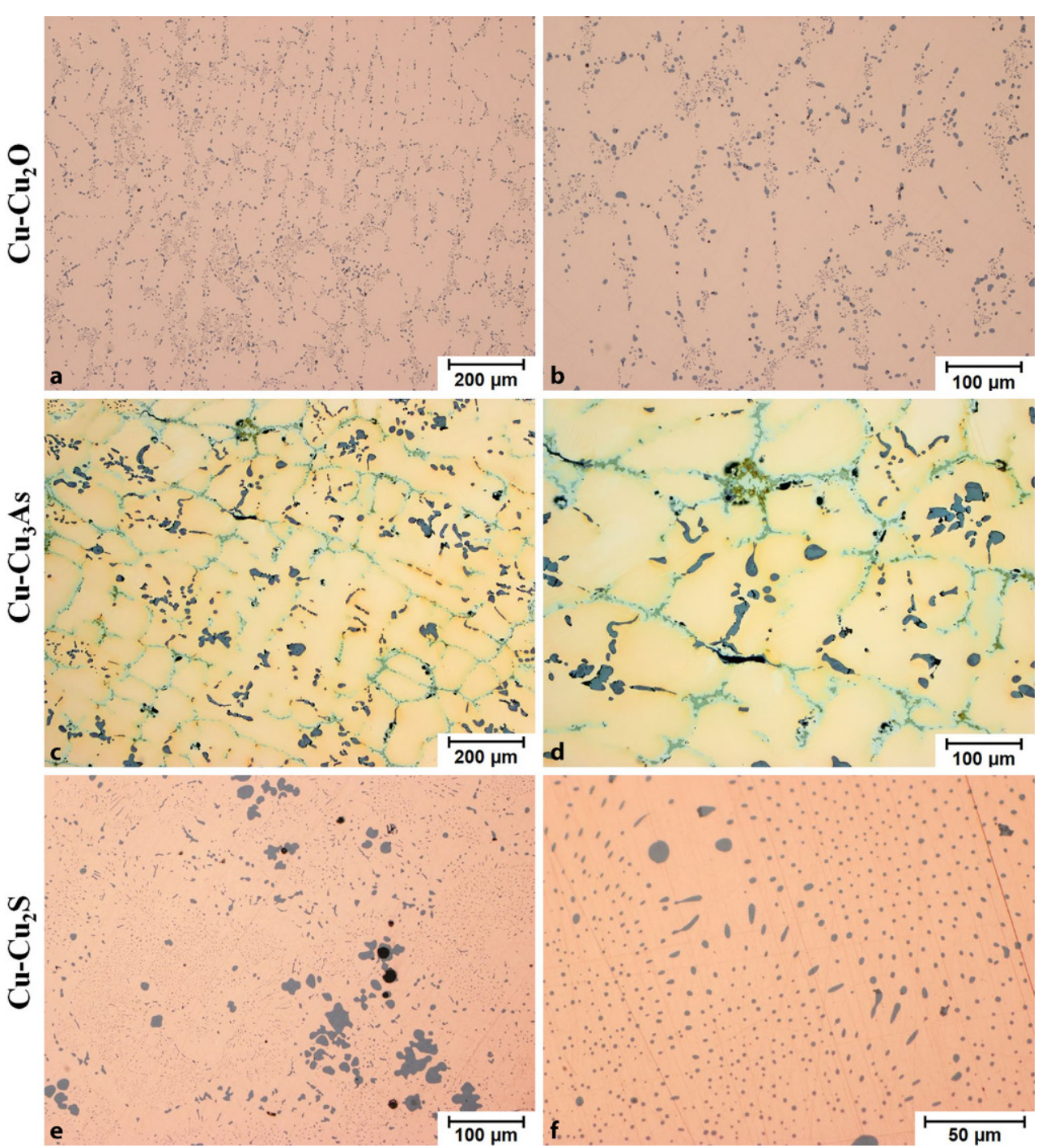

„Rösten“ notwendig, wobei durch Verbrennen der Holzkohle das Erz soweit aufgeheizt wird, dass überschüssiger Sauerstoff mit dem Erz reagieren kann. Die thermodynamischen Berechnungen zeigten, dass zuerst Schwefel zu $\mathrm{SO}_{2}$ oxidiert wird. Dabei bildet auch Arsen gasförmiges As 4 oder $\mathrm{As}_{2}$, welches beim Rösten abdampfen kann. Danach reagiert $\mathrm{Sb}$ zu flüssigem $\mathrm{Sb}_{3} \mathrm{O}_{3}$, welches aufgrund seines hohen Dampfdrucks zumindest teilweise abdampfen kann. Von den Sulfiden reagiert zuletzt $\mathrm{Cu}_{2} \mathrm{~S}$. Nur wenn gleichzeitig metallisches $\mathrm{Cu}$ und gasförmiges As vorliegen, wird $\mathrm{Cu}_{3} \mathrm{As}$ gebildet [9]. Einmal gebildetes $\mathrm{Cu}_{3} \mathrm{As}$ ist weitgehend stabil und zersetz sich erst, wenn $\mathrm{Cu}$ zu $\mathrm{Cu}_{2} \mathrm{O}$ oxidiert wird. As verdampft auch nicht während der Weiterverarbeitung durch Schmelzen und Gießen [10]. In der Praxis bedeutet dies, dass beim Rösten ein Großteil des Schwefels zu SO reagiert, $\mathrm{Cu}_{2} \mathrm{O}$ gebildet wird und Teile des $\mathrm{As}$ sowie $\mathrm{Sb}$ abdampfen. Im nachfolgenden Reduktionsschritt des $\mathrm{Cu}_{2} \mathrm{O}$ mit Kohlenstoff werden noch vorhandenes As und auch $\mathrm{Sb}$ in der Schmelze aufgenommen, und es entstehen die Arsenbronzen(Abb. 2c, d). Es ist von der Prozessführung abhängig, wieviel As in die Bronze eingebaut wird [11, 12].
Jüngste Untersuchungen legen nahe, dass Antimonbronzen durch Zusatz von Antimonerz (Antimonit) zu metallischem Kupfer hergestellt wurden [13].

Ausgehend von Chalkopyrit $\left(\mathrm{CuFeS}_{2}\right)$ wird die Metallurgie noch komplexer, und so könnten drei oder mehr Verfahrensschritte notwendig sein [14]. Wie bereits beim Einsatz von Fahlerz beschrieben, ist als erster Produktionsschritt eine Röstung erforderlich, um den Schwefelgehalt zu reduzieren. Dies erfolgte wiederum in Röstbetten, um ausreichend Sauerstoffzutritt zum Erz zu ermöglichen. In diesem Fall bilden sich zuerst $\mathrm{FeO}$ und $\mathrm{Cu}_{2} \mathrm{~S}$. Bei der Anwesenheit von Quarz kann das gebildete $\mathrm{FeO}$ zu Fayalit $\left(\mathrm{Fe}_{2} \mathrm{SiO}_{4}\right)$ weiterreagieren und so eine Schlacke bilden, wobei im Röstbett die Schmelztemperatur der Schlacke von $>1100^{\circ} \mathrm{C}$ nicht erreicht wird.

Der nächste Schritt könnte bereits ein Schmelzprozess im Schachtofen gewesen sein. Das Ergebnis dieses Prozessschrittes wird wesentlich vom vorangegangenen Röstprozess bestimmt. 

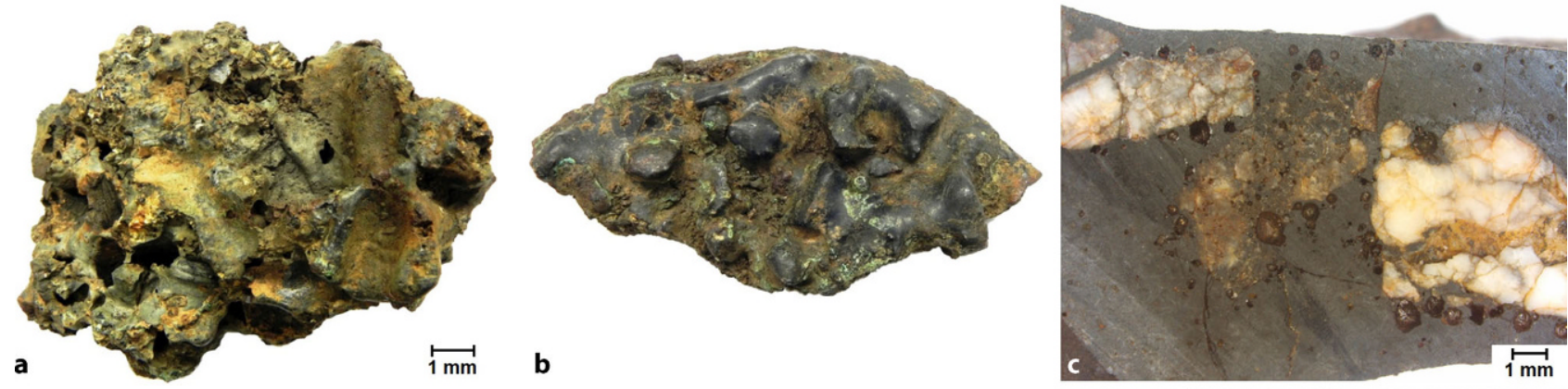

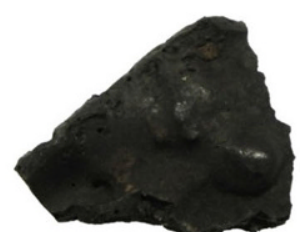

Plattenschlacke mit Rand d

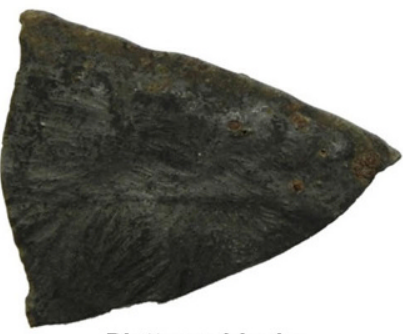

Plattenschlacke

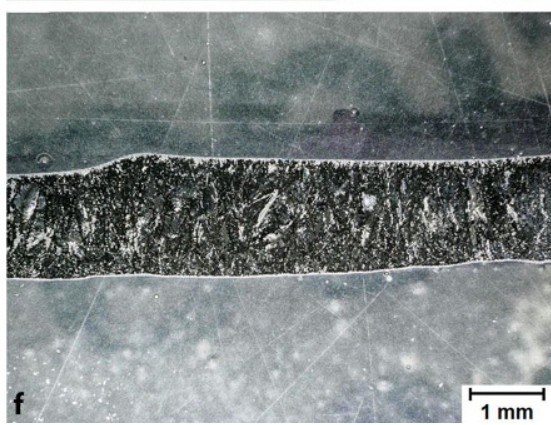

Abb. 3: Bronzezeitliche Kupferschlacken aus dem südlichen Niederösterreich: a-c Laufschlacken unterschiedlichen Aussehens c Querschnitt mit groben Quarzeinschlüssen (LOM), d-f Plattenschlacken, fDer Querschnittzeigt einen homogenen Aufbau der Schlacke (LOM)

- Falls noch viel Schwefel im Röstprodukt enthalten war, erfolgte ein Aufschmelzen der Mischung, das $\mathrm{FeO}$ verschlackte und $\mathrm{Cu}_{2} \mathrm{~S}$ bildete den sogenannten Kupferstein, auch Matte genannt. Im Kupferstein kann auch noch FeS enthalten sein. Dieser Kupferstein kann zerkleinert und abermals geröstet werden, um Schwefel zu entfernen und eine Mischung aus $\mathrm{Cu}_{2} \mathrm{O}, \mathrm{Cu}_{2} \mathrm{~S}$ und vielleicht $\mathrm{FeO}$ zu erhalten. Im nächsten Schritt erfolgt wiederum Schmelzen im Schachtofen mit Schlackenbildung und Schwarzkupfer als Endprodukt (Abb. 2e, f). Dabei reagiert $\mathrm{Cu}_{2} \mathrm{O}$ mit $\mathrm{Cu}_{2} \mathrm{~S}$ durch die sogenannte "Röstreduktionsreaktion“ zu metallischem $\mathrm{Cu}$ und $\mathrm{SO}_{2}$. Überschüssiges $\mathrm{Cu}_{2} \mathrm{O}$ kann durch den vorhandenen Kohlenstoff der Holzkohle reduziert werden.

- Wenn bereits im ersten Röstschritt der Schwefel weitgehend entfernt wurde, kann der Zwischenschritt mit Kupferstein entfallen und es entstehen direkt Schwarzkupfer und Schlacke.

Da das Gefüge des Schwarzkupfers durch das $\mathrm{Cu}-\mathrm{Cu}_{2} \mathrm{~S}$ Eutektikum geprägt ist, erscheint ein weiterer Raffinationsschritt unwahrscheinlich.

Es lässt sich zusammenfassen, dass die Metallurgie der Kupfergewinnung durch die Änderung der Erzbasis komplexer wurde und die hier angeführten Prozesse durch den Einsatz unterschiedlicher Erze oder Erzmischungen noch komplizierter wurde. Eine der Herausforderungen bei den archäometallurgischen Analysen ist es, durch die Messergebnisse auf die Erzlagerstätten die Ausgangsstoffe sowie die Herstellungsverfahren rückzuschließen.

\section{Verhüttungsschlacken und daraus abzuleitende Informationen}

Durch die Untersuchung von Schlacken erhofft man sich Erkenntnisse über die eingesetzten metallurgischen Prozesse sowie die Prozessbedingungen [15, 16]. Bei den metallurgischen Prozessen spielen Temperaturen wie Schmelz- und Erweichungspunkte eine wichtige Rolle, wobei einige Untersuchungen darauf abzielen, diese Prozessparameter zu ermitteln [17].

Rein prinzipiell ist es bei metallurgischen Prozessen erwünscht, möglichst niedrige Prozesstemperaturen zu erreichen, was bei der Kupferverarbeitung durch den Schmelzpunkt von $\mathrm{Cu}\left(1085^{\circ} \mathrm{C}\right)$ geprägt ist. Das Schmelzverhalten der Schlacke wird dabei durch ihre chemische Zusammensetzung und den gebildeten Phasen bei der Erstarrung bestimmt, wobei Schlacken der Kupferverarbeitung große Anteile an Olivin der Variante Fayalit $\left(\mathrm{Fe}_{2} \mathrm{SiO}_{4}\right)$ neben sogenannter Glasphase enthalten.

Aufgrund des Aussehens von Schlacken und deren unterschiedlichen Entstehens werden Einteilungen getroffen. Laufschlacken bezeichnen Schlacken, welche aus dem Verhüttungsofen geflossen sind (Abb. $3 a-c)$, hingegen Plattenschlacken wurden eher bei der Metallurgie in Herden und Tiegeln erhalten (Abb. 3d-f). Bei der Charakterisierung dieser Schlacken ist die Homogenität wichtig, nämlich ob die gesamte Schlacke aufgeschmolzen war (Abb. 3f) oder ob z.B. Quarzstücke in der Schlacke enthalten sind (Abb. 3c). Um Anhaltspunkte zum Schmelzverhalten der Schlacke zu erhalten, können einerseits Schmelzversuche durchgeführt und andererseits die chemische Zusammensetzung in Phasendiagrammen eingetragen werden. Um der Tatsache, dass möglichst geringe Prozesstemperaturen vorliegen sollten, gerecht zu werden, sollte man bei 

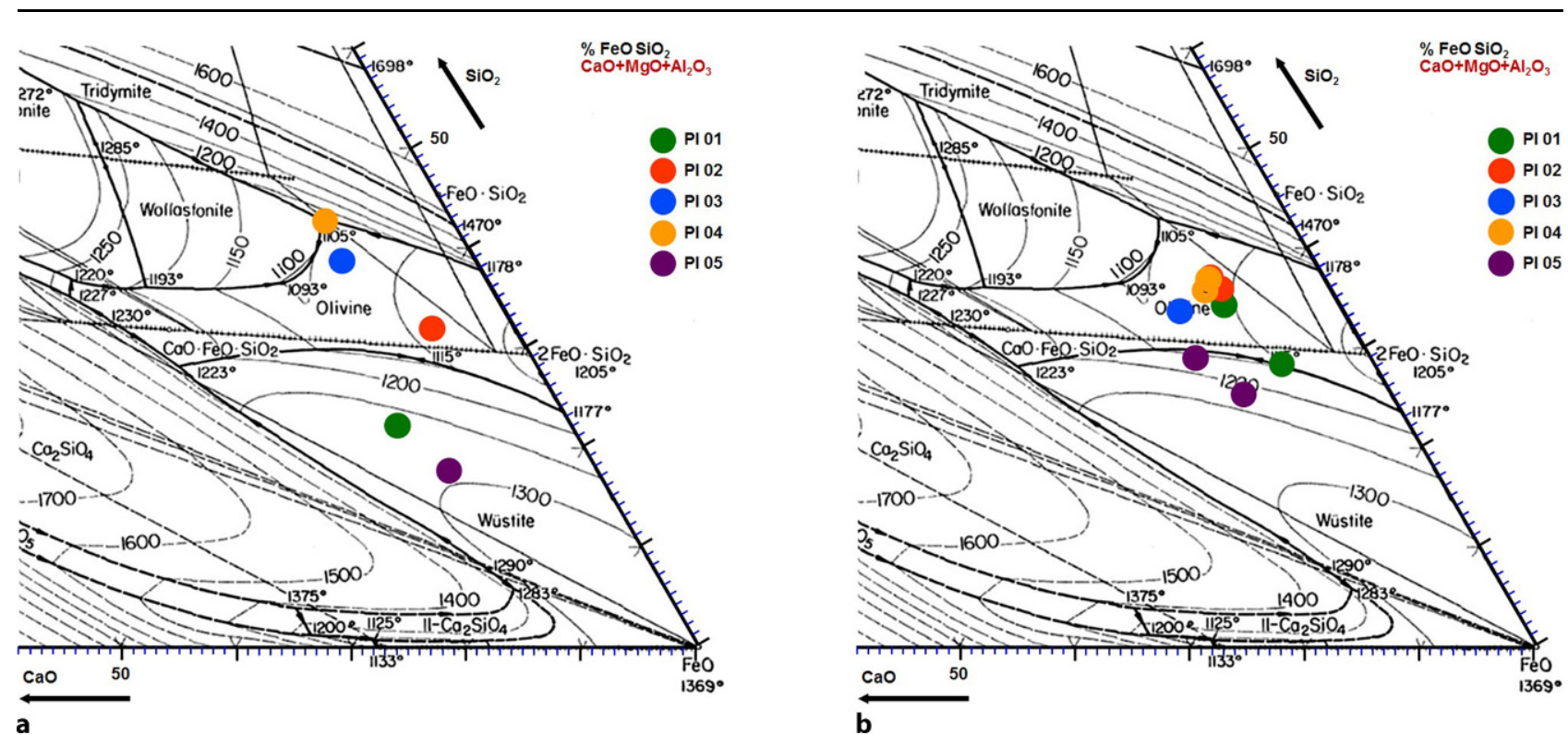

Abb. 4: Vergleich von chemischen Zusammensetzungen ausgewählter Plattenschlacken aus Prigglitz-Gasteil. Die Analysen erfolgten mittels Röntgenfluoreszenzanalyse (RFA) und Energiedispersiver Röntgenanalyse im Rasterelektronenmikroskop (REM-EDX) a RFA Messungen, b REM-EDX Messungen
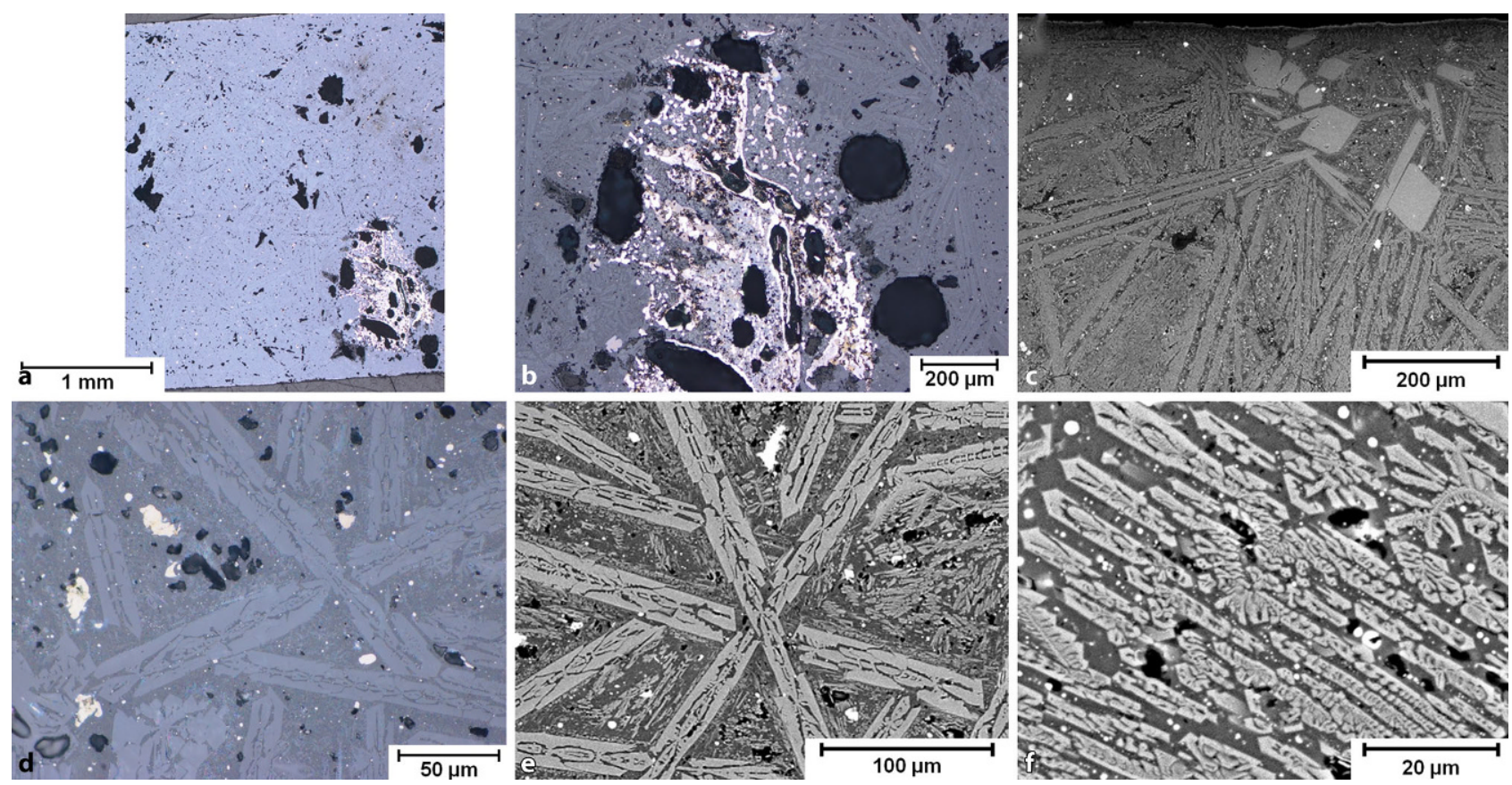

Abb. 5: Gefüge von Plattenschlacken im Lichtmikroskop und Rasterelektronenmikroskop. a Querschnitt mit Einschluss, b Detailansicht Einschluss, c Bereich mit blockigen Olivinkristallen und Fayalitbalken, d, e Fayalitbalken in Glasphase, ffeinkörniger Fayalit

Prozesstemperaturen über dem Schmelzpunkt von Fayalit $\left(1205^{\circ} \mathrm{C}\right)$ skeptisch bezüglich der Mess- oder Auswertemethodik sein.

Bei praktischen Schmelztemperaturbestimmungen (z. B. nach DIN 51730) wurden mehrfach Schmelzpunkte von $1460^{\circ} \mathrm{C}$ gemessen und auch publiziert. Da dieser Wert sehr hoch ist, stellt sich die Frage, wo der Fehler zu suchen wäre. Da die Richtigkeit der Messung außer Zweifel steht, handelt es sich offensichtlich um eine falsch eingesetzte Messmethode! Wenn Fayalit $\left(\mathrm{Fe}^{2+}\right)$ an Luft erhitzt wird, reagiert er zu Magnetit $\left(\mathrm{Fe}^{2+}, \mathrm{Fe}^{3+}\right)\left(\mathrm{Schmelzpunkt} 1535^{\circ} \mathrm{C}\right)$ oder Hämatit $\left(\mathrm{Fe}^{3+}\right)$ (Schmelzpunkt $\left.1539^{\circ} \mathrm{C}\right)$ und Quarz (Schmelzpunkt $1712^{\circ} \mathrm{C}$ ). Es wird also bei dieser Methode nicht der Schmelzpunkt der Schlacke bestimmt, sondern der Schmelzpunkt der Oxidationsprodukte.

Wird Fayalitschlacke in inerter Ar-Atmosphäre erhitzt (z. B. in einer DTA), so erfolgt keine Oxidation und Schmelzpunkte bzw. Schmelzbereiche zwischen 1100 und $1200^{\circ} \mathrm{C}$ werden gemessen [18]. 


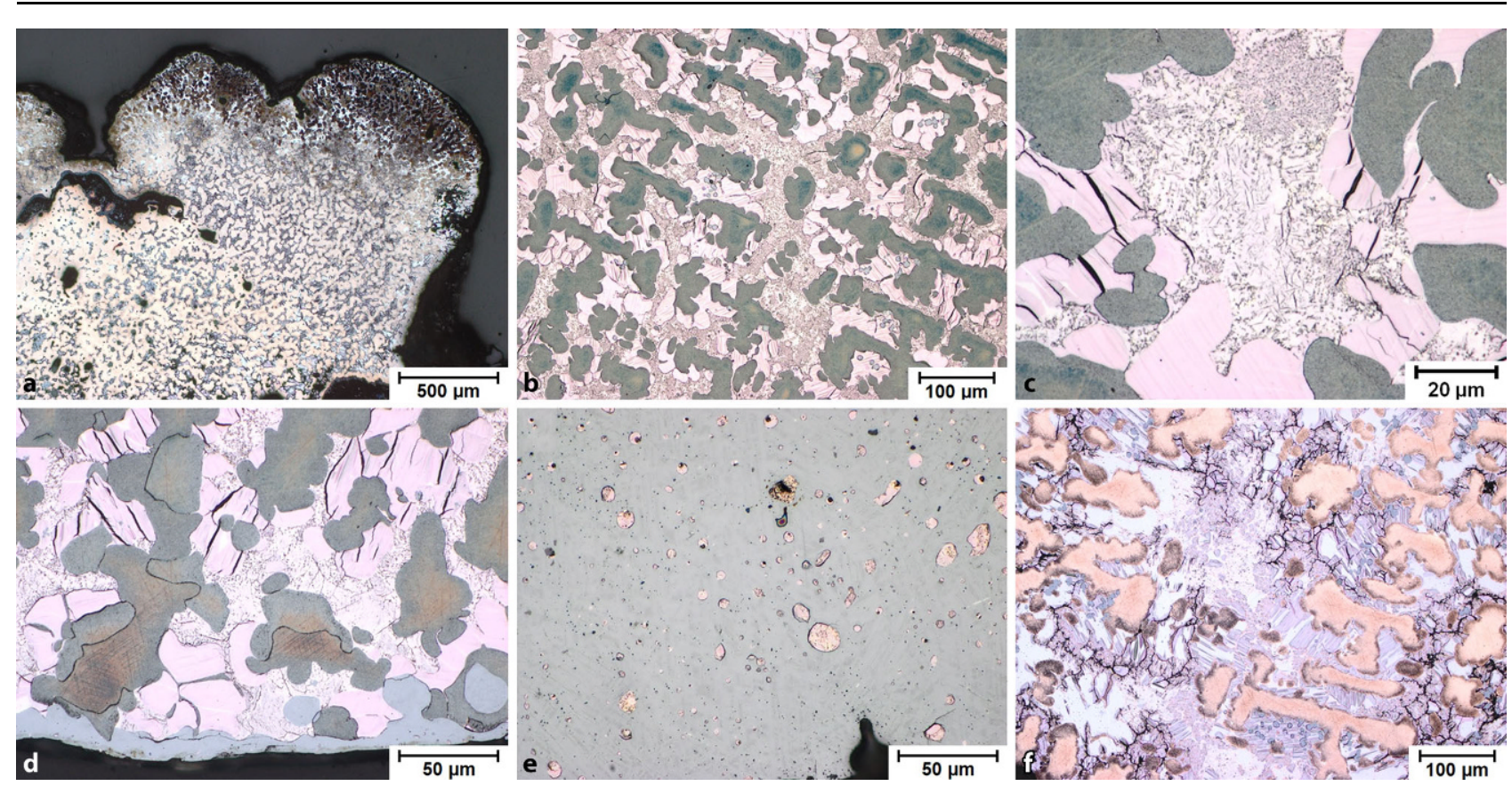

Abb. 6: Mikrostrukturen eines Gusskuchens, der durch Zusatz von Antimonit hergestellt wurde (LOM). a Übersicht eines Randbereichs, b-f die unterschiedlichen Gefüge zeigen, dass bei einer derartigen Probe eine einzelne Punktmessung nicht aussagekräftig ist

Eine beliebte Methode, Schlackenschmelzpunkte zu ermitteln, sind chemische Analysen und die Verwendung von Phasendiagrammen, in welche die gemessenen Elementkonzentrationen eingetragen werden (Abb. 4; [19]). Zwei Punkte sind hier zu beachten, nämlich erstens ob das verwendete Phasendiagramm geeignet ist, um relevante Aussagen bezüglich des Schmelzverhaltens der Schlacke machen zu können, und zweitens wie homogen das analysierte Schlackenvolumen war. Abb. 4 zeigt Vergleiche von Röntgenfluoreszenzanalysen (RFA) (großes analytisches Volumen) (Abb. 4a) und Energiedispersiever Röntgenanalyse im Rasterelektronenmikroskop (REM-EDX) (kleines analytisches Volumen) (Abb. 4b).

Um Schlacken wirklich beurteilen zu können, scheint eine metallographische Gefügeanalyse mit REM-EDX Auswertung unumgänglich. Nur so kann sichergestellt werden, dass im analysierten Bereich keine groben Inhomogenitäten vorliegen (Abb. $5 \mathrm{a}, \mathrm{b}$ ) und welche Morphologie die vorliegenden Phasen haben (Abb. 5c-f). Üblicherweise liegen in Plattenschlacken Mischungen aus Olivinen, Wüstit (FeO) und Glasphase vor, wobei die Glasphase die restlichen in der Schlacke vorliegenden Elemente enthält.

Es sollte aber darauf hingewiesen werden, dass aufgrund der Komplexität der Schlackenchemie aus Einzeloder auch Kleinserienanalysen keine universellen Antworten abgeleitet werden können. Die Zusammensetzung der Schlacke wird vom Erz, von den metallurgischen Prozessen, von den verwendeten Ofenmaterialien sowie Brennstoffen bestimmt, womit für Detailaussagen das „Kaffeesudlesen“ vermutlich exaktere Aussagen liefert.

\section{Bronzezeitliche Kupferlegierungen}

Bei den bronzezeitlichen Kupferlegierungen können grob 4 Gruppen unterschieden werden.

- Nahezu reines Kupfer, welches direkt nach dem Gewinnungs- bzw. Raffinationsprozess verwendet wurde. Dazu zählt Kupfer aus oxydischen Erzen mit $\mathrm{Cu}_{-} \mathrm{Cu}_{2} \mathrm{O}$ Eutektikum, aber auch Kupfer aus der Verhüttung von Chalkopyrit mit $\mathrm{Cu}-\mathrm{Cu}_{2} \mathrm{~S}$ Eutektikum. Die mechanischen Eigenschaften dieser Kupfergegenstände waren nicht sehr gut, aber die Härte von Werkstücken konnte durch Verformung (z. B. Hämmern) verbessert werden.

- Kupfer aus Fahlerzen, in dem As und/oder Sb durch Mischkristallhärtung oder die Bildung intermetallischer Phasen eine Verbesserung der mechanischen Eigenschaften bewirkt. Die Konzentrationen an Legierungselementen werden dabei im Wesentlichen durch die Prozessführung bei der Verhüttung bestimmt (Abb. 6; $[12,13])$.

- Zinnbronzen, bei denen absichtlich Legierungselemente zugesetzt werden, zeigen ebenfalls eine Verbesserung der mechanischen Eigenschaften durch Mischkristallhärtung oder Bildung intermetallischer Phasen. Ob das Zinn in metallischer Form oder als Zinnstein $\left(\mathrm{SnO}_{2}\right)$ zugesetzt wurde, ist in den meisten Fällen noch nicht geklärt. Zusätzliche Schwankungen in den Legierungszusammensetzungen entstehen durch die Verwendung der verschieden hergestellten Kupferqualitäten (Abb. 7; $[20,21])$.

- Recyclingkupfer wird, wie der Name vermuten lässt, durch Verschmelzen bereits verwendeter Kupferteile erhalten. In diesem Fall erhält man Mischungen der 3 bereits beschriebenen Kupferlegierungen, und da- 
Abb. 7: Griffzungenmesser. a Entnahme eines Keils für die Analyse. b Übersichtsaufnahme mit einer Patina, $\mathbf{c}, \mathbf{d}$ Gefüge mit $\mathrm{Cu}_{2} \mathrm{~S}$ sowie $\mathrm{Cu}_{41} \mathrm{Sn}_{11}$ Ausscheidungen, e Cu-Sn Eutektoid aus $\mathrm{Cu}_{41} \mathrm{Sn}_{11}$ und $\mathrm{Cu}-\mathrm{Sn}$ Mischkristall, $\mathrm{f}$ Patina an der Metalloberfläche
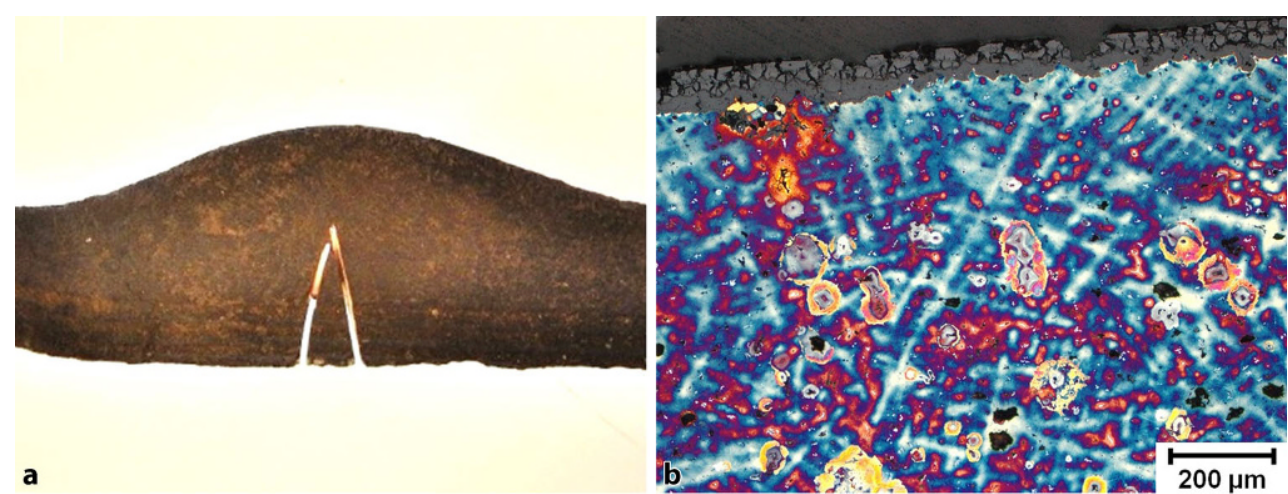

a
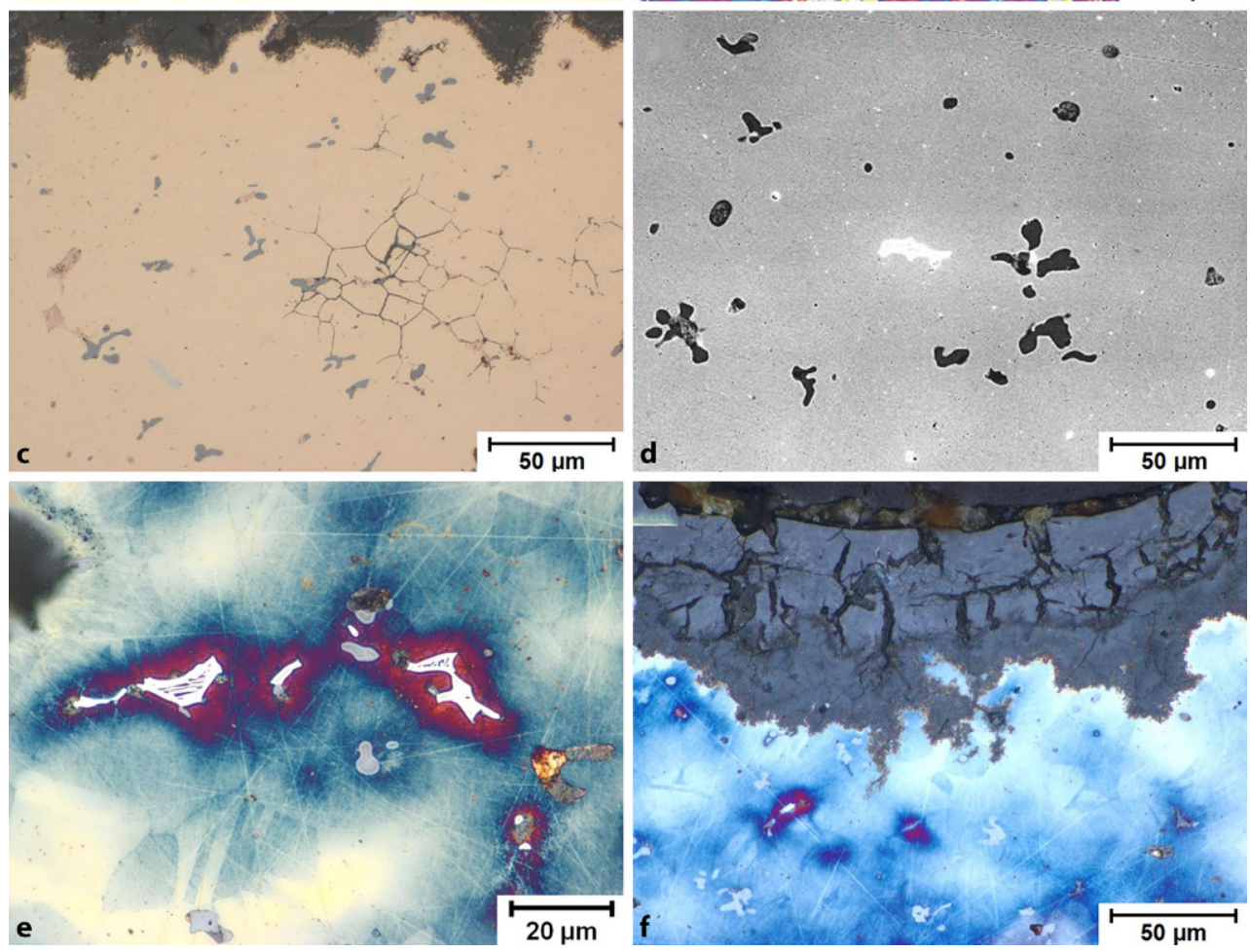

mit werden Aussagen über Herkunft der Erze und die Metallurgie der Kupfergewinnung unmöglich.

Metallurgischen Untersuchungen von archäologischen Kupfergegenständen steht man üblicherweise kritisch gegenüber, vor allem was eine repräsentative Probennahme betrifft. Als Werkstoffwissenschaftler würden wir einen Querschnitt mit anschließender metallographischer Präparation empfehlen, was zur partiellen Zerstörung des Gegenstandes führen würde und daher umgehend abgelehnt wird. Bei entsprechendem Interesse dürfen Oberflächlenanalysen durchgeführt werden oder die Entnahme kleiner Keile wird erlaubt (Abb. 7a), wobei ein Restaurator das ursprüngliche Aussehen möglichst wiederherstellt.

In beiden Fällen ist aber zu berücksichtigen, dass die analysierten Proben nicht die Zusammensetzung und das Gefüge des gesamten Objekts widerspiegeln. Insbesondre bei Oberflächenanalysen wäre zu beachten, dass bereits bei der Erstarrung der Schmelze aufgrund von Seigerungen unter- schiedliche Legierungskonzentrationen vorliegen können. Zusätzlich wären noch Veränderungen der Elementkonzentrationen durch Korrosion zu erwähnen (Abb. 8; [22]).

Bei der Beschreibung von Gefügen der Kupferlegierungen sollte man sich weniger an den "narrativen" Charakter der Archäologie, sondern mehr an die naturwissenschaftlichen Begriffe und Erklärungen halten. Auch diesbezüglich möchte ich auf negative Literaturbelege verzichten.

Es darf nicht unerwähnt bleiben, dass die metallographischen Untersuchungen von Legierungen nur eher selten für die Beurteilung von Kupferobjekten herangezogen werden, da derartige Messungen relativ zeitaufwändig sind. Daher wird versucht, durch Spurenanalysen von Begleitelementen und Messung von Isotopenverhältnissen, an möglichst kleinen Probenmengen, Rückschlüsse auf die angewandte Metallurgie zu ziehen. Die "Zahlenberge" (Analysendaten) werden immer größer, aber aufgrund der schlechten Vergleichbarkeit der Werte bezüglich Probennahme und Vorgeschichte ist es sehr schwierig, Korrelationen zu erhalten. 
Abb. 8: Korrodierter Kupfertropfen im LOM, a die Übersichtsaufnahme zeigt die langen Kupferdendriten welche zuerst erstarrt sind, sowie die bevorzugte Korrosion der interdendritischen Bereiche, b-d Detailaufnahmen, d Verwendung von polarisiertem Licht
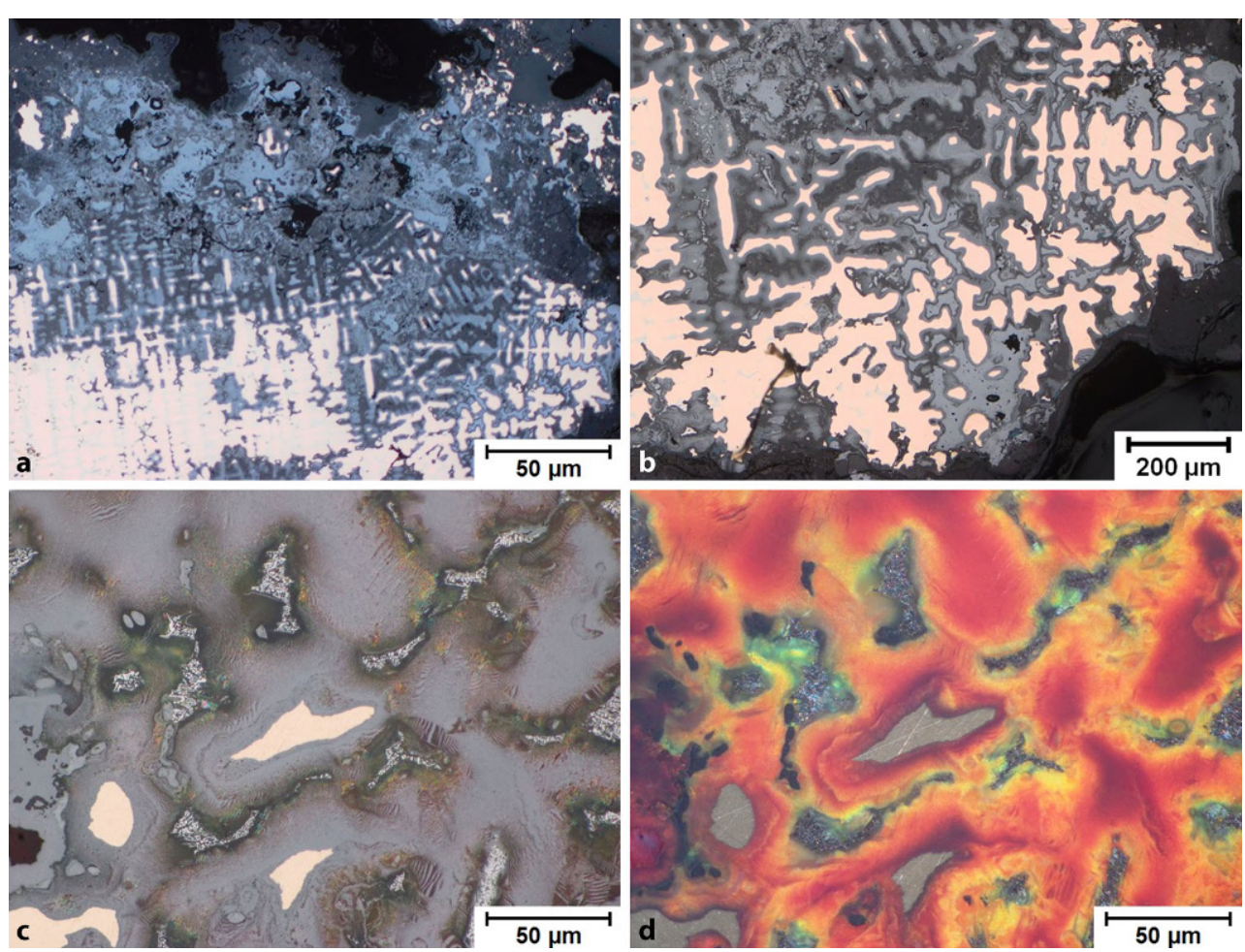

Für die Zukunft der Archäometallurgie erscheint es uns sehr wichtig, dass eine klare Differenzierung zwischen "narrativer" Archäologie und "naturwissenschaftlicher" Metallurgie erfolgt. Eine "narrative“ Metallurgie bedeutet einen Rückschritt in die mittelalterliche Alchemie [23].

\section{Zusammenfassung}

Die bronzezeitliche Verhüttung von Kupfer in den Ostalpen ist durch zahlreiche Schmelzplätze belegt, was die Bedeutung der Kupfergewinnung in dieser Region klar aufzeigt.

Ziel der Archäometallurgie ist es nun, durch die naturwissenschaftliche Untersuchung von Schlacken und Kupfergegenständen Rückschlüsse auf die verwendeten Verhüttungsprozesse zu erlangen.

Eine erste Voraussetzung dafür ist, dass Untersuchungsmethoden eingesetzt werden, deren Ergebnis die Beantwortung der Fragestellung erlaubt (z.B. Bestimmung der Schmelztemperatur von Plattenschlacken).

Eine zweite Voraussetzung wäre ein homogenes Probenmaterial. Schlacken für die Analyse sollten vollständig aufgeschmolzen gewesen sein und keine groben Quarzeinschlüsse enthalten. Lokale Unterschiede in Legierungen aufgrund von Seigerungen bei der Erstarrung oder Korrosionserscheinungen - verursachen ebenfalls Inhomogenitäten.

Falls diese beiden Voraussetzungen nicht erfüllt sein sollten, was in den meisten Fällen auch der Fall ist, muss man sich dessen Bewusst sein und dies bei der Auswertung der Analysenergebnisse berücksichtigen. Vor allem ist Skepsis angebracht, wenn Untersuchungsergebnisse nicht den naturwissenschaftlichen Grundlagen entsprechen.
Naturwissenschaftliche Untersuchungen sind notwendig, um prähistorische Verhüttungsmethoden zu erklären, wobei für die Datenauswertung der "narrative“ Charakter der Archäologie im Hintergrund und der "naturwissenschaftliche" Charakter der Metallurgie im Vordergrund stehen muss.

Als "narrative" Beschreibung betrachte ich auch, wenn den bronzezeitlichen Metallurgen unterstellt wird, dass sie wussten, ob im Kupfer z. B. Schwefel oder Sauerstoff enthalten war und sie entsprechend darauf reagierten.

Danksagung. Mein Dank gilt meiner Kollegin Frau Dr. Susanne Strobl für ihre hervorragenden metallographischen Probenpräparationen, die jede Publikation auch zu einem optischen Erlebnis machen. Weiters danke ich den Herren Wolfgang Scheiblechner (Kunstschmied in Palfau), Dr. Hannes Herdits (Burgenländische Landesmuseen) und Prof. Peter Trebsche (Universität Innsbruck) für zahlreiche Diskussionen über Metallurgie und Archäologie sowie umfangreiches Probenmaterial.

Funding. Open access funding provided by TU Wien (TUW).

Interessenkonflikt. R. Haubner gibt an, dass kein Interessenkonflikt besteht.

Open Access Dieser Artikel wird unter der Creative Commons Namensnennung 4.0 International Lizenz veröffentlicht, welche die Nutzung, Vervielfältigung, Bearbeitung, Verbreitung und Wiedergabe in jeglichem Medium und Format erlaubt, sofern Sie den/die ursprünglichen Autor(en) und die Quelle ordnungsgemäß nennen, einen Link zur Creative Commons Lizenz beifügen und angeben, ob Änderungen vorgenommen wurden.

Die in diesem Artikel enthaltenen Bilder und sonstiges Drittmaterial unterliegen ebenfalls der genannten Creative Commons Lizenz, sofern sich aus der Abbildungslegende nichts anderes ergibt. Sofern das betreffende Material nicht unter der genannten Creative Commons Lizenz steht und die betreffende Handlung nicht nach gesetzlichen Vorschriften erlaubt 
ist, ist für die oben aufgeführten Weiterverwendungen des Materials die Einwilligung des jeweiligen Rechteinhabers einzuholen.

Weitere Details zur Lizenz entnehmen Sie bitte der Lizenzinformation auf http://creativecommons.org/licenses/by/4.0/deed.de.

\section{Literatur}

1. Tylecote, R. F.; Ghaznavi, H. A.; Boydell, P. J.: Partitioning of Trace Elements between the Ores, Fluxes, Slags and Metal during the Smelting of Copper, Journal of Archaeological Science, 4 (1977), pp 305-333

2. Tylecote, R. F: A history of metallurgy, The Metals Society, London: Mid County Press London, 1976

3. Tylecote, R. F.: Summary of results of experimental work on early copper smelting, in Oddy, W. A. (ed.): Aspects of Early Metallurgy, London: British Museum, 1980, pp 5-12

4. Tylecote, R. F.: The early history of metallurgy, London-New York: Longman, 1987

5. Pernicka, E.; Lutz, J.; Stöllner, T.: Bronze age copper produced at Mitterberg, Austria, and its distribution, Archaeologia Austriaca, by Österreichische Akademie der Wissenschaften, Wien, 100 (2016), pp 19-55

6. Haubner, R.; Strobl, S.; Thurner, M., Herdits, H.: Ein Metallflachbeil der Kupferzeit aus Bernstein, Burgenland, Berg- und Hüttenmännische Monatshefte, 165 (2020) S. 447-452

7. Haubner, R.; Strobl, S.: Metallographische Untersuchung eines Kupferflachbeils aus der Kupferzeit, in Neidel, A. (Hrsg.): Sonderbände der Praktischen Metallographie zur 54. Metallographie Tagung, 2020, S. 202-207

8. Sperl, G.: Der Werkstoff des „Ötzibeiles" Die kupferzeitliche Beilklinge der Gletschermumie aus den ÖtztalerAlpen, BHM Berg- und Hüttenmännische Monatshefte, 163 (2018), S. 451-455

9. Haubner, R.; Strobl, S.: Consideration about the metallurgy of $\mathrm{Cu}$ As Bronzes, in: Monographies Instrumentum, submitted 2020

10. Mödlinger, M.; Oro Calderon, R.; Haubner, R.: Arsenic loss during metallurgical processing of arsenical bronze, Archaeol Anthropol Sci, 11 (2019), pp 133-140

11. Ertl, F.; Strobl, S.; Haubner, R.: An ancient bronze ingot smelted from fahlore, Materials Science Forum, 891 (2017), pp 613-617

12. Haubner, R.; Ertl, F.; Strobl, S.: Examinations of a Bronze Ingot Made of Fahlore, Practical Metallography, 54 (2017), pp 107-117

13. Haubner, R., Strobl, S., Thurner, M., Herdits, H.: Ein Kupfergusskuchen mit hohem Antimongehalt aus Velem/Westungarn, Berg und Huettenmännische Monatshefte, 165 (2020), Nr. 9, S. 453-460
14. Herdits, H.: Die ostalpine bronzezeitliche Kupfererzeugung im überregionalen Vergleich am Grundbeispiel eines Hüttenplatzes in Mühlbach/Sbg., Dissertation an der Universität Wien, Wien, 2017

15. Haubner, R.; Strobl, S.: Slag investigation from iron smelting and iron processing sites in Austria-from Hallstatt and medieval period and the 19th century, Materials Science Forum, 782 (2014), pp 635-640

16. Haubner, R.; Strobl, S.; Klemm, S.; Trebsche, P.: Prähistorische Kupfergewinnung im südöstlichen Niederösterreich - archäometallurgische Untersuchungen an alten und neuen Fundstücken, in Lauermann, E.; Trebsche, P. (Hrsg.): Beiträge zum Tag der Niederösterreichischen Landesarchäologie 2015, Niederösterreichisches Landesmuseum, Asparn/Zaya, 2015, S. 26-33.

17. Slag Atlas, 2nd Edition, Edited by Verein Deutscher Eisenhüttenleute (VDEh), Düsseldorf: Verlag Stahleisen $\mathrm{GmbH}, 1995$

18. Klemm, S.; Strobl, S.; Haubner, R.: Mediaeval Iron Smelting in the Area of the Iron Mountain (Steirischer Erzberg) at Eisenerz, Styria (Austria), in Forschungszentrum HiMAT (Hrsg.): Mining in European History and its Impact on Environment and Human Societies, Innsbruck: innsbruck university press, 2013, pp 103-109

19. Haubner, R.; Strobl, S.; Klemm, S.: Investigations of a slag from copper smelting discovered at the Bronze Age site Prein VII/Cu in Lower Austria, in Montero-Ruiz, I.; Perra, A. (eds.): Archaeometallurgy in Europe IV, Bibliotheca Praehistorica Hispana, Vol. 33. Editorial CSIC, Madrid, 2017, pp 135-142

20. Haubner, R.; Strobl, S.; Trebsche, P.: Analysis of Urnfield period bronze droplets formed during casting, Materials Science Forum, 891 (2017), pp 41-48

21. Haubner, R.; Strobl, S.; Trebsche, P.: Metallographic analyses from the late Urnfield period copper mining settlement at Prigglitz-Gasteil in Lower Austria, in Turck, R.; Stöllner, T.: Goldenberg, G. (eds.): Alpine Copper II-Alpenkupfer II-Rame delle Alpi II-Cuivre des Alpes II. New Results and Perspectives on Prehistoric Copper Production, Der Anschnitt, Beiheft, 42 (2019) pp 323-332

22. Haubner, R.; Strobl, S.: Long-time corrosion of a cast bronze droplet during 3000 years storage in soil, in Proceedings EUROCORR 2015, Paper-Nr. 116 (2015) pp 1-6

23. Khunrath, H.: Vom hylealischen, das ist / pri-materialischen catholischen oder allgemeinen natürlichen Chaos, der naturgemässen Alchymiae und Alchymisten, Ausgabe Frankfurt, 1708

Hinweis des Verlags. Der Verlag bleibt in Hinblick auf geografische Zuordnungen und Gebietsbezeichnungen in veröffentlichten Karten und Institutsadressen neutral. 